\title{
Advective Flow of a Rotating Fluid Layer in a Vibrational Field
}

\author{
K. G. Shvarts
}

This paper presents a derivation of new exact solutions to the Navier-Stokes equations in Boussinesq approximation describing two advective flows in a rotating thin horizontal fluid layer with no-slip or free boundaries in a vibrational field. The layer rotates at a constant angular velocity; the axis of rotation is aligned with the vertical axis of coordinates. The temperature is linear along the boundaries of the layer. The case of longitudinal vibration is considered. The resulting solutions are similar to those describing the advective flows in a rotating fluid layer with solid or free boundaries without vibration. In both cases, the velocity profile is antisymmetric. Thus, in particular, in the absence of rotation, the longitudinal vibration in the presence of advection can be considered as a kind of "one-dimensional" rotation. The presence of rotation initiates the vortex motion of the fluid in the layer. Longitudinal vibration has a stronger effect on the $x$ th component of the velocity than on the $y$ th component. At large values of the Taylor number and (or) the vibration analogue of the Rayleigh number thin boundary layers of velocity, temperature and amplitude of the pulsating velocity component arise, the thickness of which is proportional to the root of the fourth degree from the sum of these numbers.

Keywords: horizontal convection, longitudinal vibration, exact solution

\section{Introduction}

Advective flows occur in a horizontal layer of an incompressible fluid with a horizontal temperature gradient at its boundaries causing horizontal convection. In the case of linear temperature distribution, the flow is described analytically, being an exact solution of the Navier - Stokes equations in Boussinesq approximation [1, 17].

Received July 09, 2019

Accepted August 19, 2019

Konstantin G. Shvarts

kosch@psu.ru

Perm State University

ul. Bukireva 15, Perm, 614990 Russia

RUSSIAN JOURNAL OF NONLINEAR DYNAMICS, 2019, 15(3), 261-270 
In [11] G. Z. Gershuni and E. M. Zhukhovitsky deduce a formula describing plane-parallel advective flow in an infinite horizontal layer at vibration. In $[7,8]$ it was shown that vibration increases the stability of advective flow under gravity conditions of practically all types of disturbances, excluding flat thermal waves, whose existence area moves towards small values of the Prandtl number. In [9] R. V. Birikh described advective flow that occurs in a zero-gravity situation under the action of linear high-frequency oscillations, and the hydrodynamic instability of this flow is studied in [2]. In $[15,16]$ the advective flow, which is formed in a vertical magnetic field, has a velocity profile similar to the flow profile in a vibration field [11]. The paper [3] presents a new class of exact solutions describing advective flows in a horizontal fluid layer with nonlinear temperature distribution at the boundaries. The study of vibration effects in solids is presented in $[6,10]$.

The monograph [4] presents a procedure for obtaining exact solutions of Navier-Stokes equations describing closed advective flows in a rotating horizontal layer of incompressible fluid. On its basis, the new solutions describing the advective flow in a rotating layer in a vibration field are constructed.

\section{Exact solution}

Let us consider a thin infinite horizontal layer of an incompressible fluid with flat boundaries $z= \pm h$ that have a linear temperature distribution along the axis $O x$ (Fig. 1). The layer rotates at a constant angular velocity $\Omega_{0}$, and the axis of rotation is aligned with the vertical axis of coordinates $O z$. Let us consider the situation where the Froude number $F r=\Omega_{0}^{2} l / g \ll 1[12]$, $l$ being a characteristic horizontal scale, and $g$ the acceleration of gravity. Following [13, 14], the dimensionless equations for averaged velocity and temperature fields in the presence of vibration are written in a rotating coordinate system. By choosing as the units of length $x, y, z$, time $t$, velocity $\vec{v}=\left(v_{x}, v_{y}, v_{z}\right)$, temperature $T$ and pressure $P$, respectively, the half-thickness of the layer $h, h^{2} / \nu, g \beta A h^{2} / \nu, A h, \rho_{0} g \beta A h^{2}$ (here $\nu$ is the kinematic viscosity, $\beta$ is the coefficient of thermal expansion, $\rho_{0}$ is the average density, $A$ is a constant horizontal temperature gradient at the boundaries of the layer), the initial equations in dimensionless form have the following representation:

$$
\begin{gathered}
\frac{\partial \vec{v}}{\partial t}+G r(\vec{v} \nabla) \vec{v}+\sqrt{T a}\left(\overrightarrow{i_{z}} \times \vec{v}\right)=-\nabla P+\Delta \vec{v}+T \overrightarrow{i_{z}}+\frac{R a_{V}}{G r P r}(\vec{w} \nabla)(T \vec{n}-\vec{w}), \\
\operatorname{div} \vec{v}=0, \quad \operatorname{div} \vec{w}=0, \quad \operatorname{rot} \vec{w}=\nabla T \times \vec{n}, \\
\frac{\partial T}{\partial t}+G r \vec{v} \nabla T=\frac{1}{P r} \Delta T,
\end{gathered}
$$

where $\vec{w}$ is the amplitude of the pulsating component of velocity, (its unit here coincides with the unit of temperature measurement), $\vec{n}=\left(n_{x}, n_{y}, n_{z}\right)$ is the axis of vibration, $\vec{i}_{z}=(0,0,1)$, $T a=\left(2 \Omega_{0} h^{2} / \nu\right)^{2}$ is the Taylor number, $G r=g \beta A h^{4} / \nu^{2}$ is the Grashof number, $\operatorname{Pr}=\nu / \chi$ is the Prandtl number, where $\chi$ is the coefficient of thermal diffusivity, $R a_{v}=\left(\beta b \omega A h^{2}\right)^{2} / 2 \nu \chi$ is the vibrational analogue of the Rayleigh number (the Gershuni number), $b$ is the amplitude of displacement, $\omega$ is the circular frequency of harmonic oscillations in the gravity field, performed by non-isothermal fluid layer, the Laplace operator $\Delta \equiv \partial^{2} / \partial x^{2}+\partial^{2} / \partial y^{2}+\partial^{2} / \partial z^{2}$, $\nabla$ is a gradient.

Following the conclusions of [11], we restrict ourselves to the case of longitudinal vibration: $\vec{n}=(1,0,0)$. In a flat rotating layer of an incompressible fluid, a stationary advective flow 


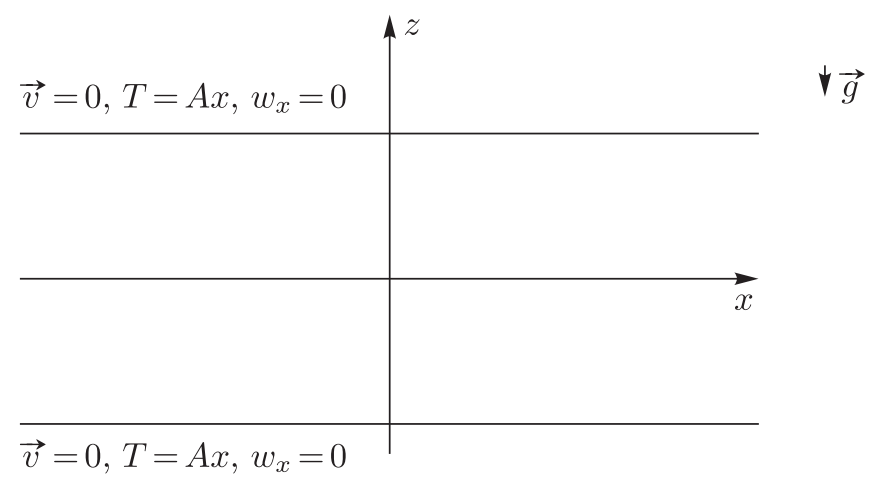

Fig. 1. A sketch of the geometry of the infinite horizontal fluid layer for the case of solid boundaries.

homogeneous in the plane $x, y$ is formed:

$$
\begin{gathered}
v_{x}=u_{0}(z), \quad v_{y}=v_{0}(z), \quad v_{z}=0, \quad T=x+\tau_{0}(z), \quad P=p_{0}(x, y, z), \\
w_{x}=w_{x}(z), \quad w_{y}=w_{z}=0 .
\end{gathered}
$$

Two problems with symmetric boundary conditions will be considered which will allow us to find an exact solution quite easily. It is a rotating horizontal layer with no-slip and with free boundaries.

\subsection{A layer with solid boundaries}

On the horizontal boundaries of the layer at $z= \pm 1$ :

$$
\vec{v}=0, \quad T=x, \quad w_{x}=0 .
$$

Substituting Eq. (2.4) into the system of equations (3.1)-(2.3), (2.5), we obtain the equations for finding the velocity, temperature and pressure:

$$
\begin{gathered}
\frac{\partial p_{0}}{\partial z}=T, \quad-\sqrt{\operatorname{Ta}} v_{0}=-\frac{\partial p_{0}}{\partial x}+u_{0}^{\prime \prime}+\frac{R a_{V}}{G r P r} w_{x}, \\
\sqrt{\operatorname{Ta}} u_{0}=-\frac{\partial p_{0}}{\partial y}+v_{0}^{\prime \prime}, \quad G r u_{0} \frac{\partial T}{\partial x}=\frac{1}{\operatorname{Pr}} \tau_{0}^{\prime \prime}, \quad w_{x}^{\prime}=\frac{\partial T}{\partial z} .
\end{gathered}
$$

Let us add boundary conditions

$$
u_{0}( \pm 1)=0, \quad v_{0}( \pm 1)=0, \quad \tau_{0}( \pm 1)=0, \quad w_{x}( \pm 1)=0
$$

and closed-loop conditions

$$
\int_{-1}^{1} u_{0} d z=0, \quad \int_{-1}^{1} v_{0} d z=0
$$

and let us start looking for an exact solution to this problem.

The first equation of the system (2.6) is integrated to determine the pressure:

$$
p_{0}=p_{h}+x(z+1)+\int_{-1}^{z} \tau_{0}(\zeta) d \zeta
$$


where $p_{h}$ is the pressure at the lower boundary. The velocity, temperature and the amplitude of the pulsating component of velocity are obtained by substituting Eq. (2.9) into Eqs. (2.6)

$$
\begin{gathered}
u_{0}^{\prime \prime}(z)+\sqrt{T a} v_{0}(z)=\frac{\partial p_{h}}{\partial x}+(z+1)-\frac{R a_{V}}{\operatorname{GrPr}} w_{x}(x), \\
v_{0}^{\prime \prime}(z)-\sqrt{T a} u_{0}(z)=\frac{\partial p_{h}}{\partial y}, \\
\tau_{0}^{\prime \prime}(z)=\operatorname{Rau}_{0}(z), \quad w_{x}^{\prime}=\tau_{0}^{\prime}(z) .
\end{gathered}
$$

With the help of the first equation of $(2.10) v_{0}(z)$ is defined as $v_{0}(z)=\frac{1}{\sqrt{T a}}\left[\frac{\partial p_{h}}{\partial x}+(z+1)-\right.$ $\left.-u_{0}^{\prime \prime}(z)-\frac{R a_{V}}{\operatorname{GrPr}} w_{x}(x)\right]$, then from the second equation $\sqrt{\operatorname{Ta}} u_{0}(z)=-\frac{\partial p_{h}}{\partial y}+\frac{1}{\sqrt{\operatorname{Ta}}}\left[-u_{0}^{I V}(z)-\right.$ $\left.-\frac{R a_{V}}{G r P r} w_{x}^{\prime \prime}(z)\right]$, in addition, $w_{x}^{\prime \prime}=\tau_{0}^{\prime \prime}=\operatorname{GrPr} u_{0}(z)$. As a result, to find the first component of the velocity, an ordinary differential equation of the fourth order is found, which has the form

$$
u^{I V}(z)+\left(T a+R a_{V}\right) u_{0}(z)+\sqrt{T a} \frac{\partial p_{h}}{\partial y}=0 .
$$

Taking into account the boundary conditions (2.7), we obtain

$$
u_{0}( \pm 1)=0, \quad u_{0}^{\prime \prime}(-1)=\frac{\partial p_{h}}{\partial x}, \quad u_{0}^{\prime \prime}(1)=\frac{\partial p_{h}}{\partial x}+2 .
$$

From the condition of flow closure (2.8) $\frac{\partial p_{h}}{\partial y}=0$ and $\frac{\partial p_{h}}{\partial x}+1=0$.

The second component of the velocity $v_{0}(z)$ is found using the third equation of (2.6) and the corresponding conditions (2.7) and (2.8).

The temperature component $\tau_{0}(z)$ is based on the boundary-value problem

$$
\tau_{0}^{\prime \prime}(z)=\operatorname{GrPru} u_{0}(z), \quad \tau_{0}( \pm 1)=0 .
$$

The solution of the problem (2.11)-(2.13) subject to condition (2.8) has the following representation:

$$
\begin{gathered}
u_{0}(z)=\frac{1}{\sqrt{T a+R a_{V}}} \operatorname{Im} f_{1}(z), \quad v_{0}(z)=\frac{\sqrt{T a}}{T a+R a_{V}}\left[z-\operatorname{Re} f_{1}(z)\right], \\
\tau_{0}(z)=w_{x}(z)=\frac{G r P r}{T a+R a_{V}}\left[z-\operatorname{Re} f_{1}(z)\right],
\end{gathered}
$$

where $f_{1}(z)=\sinh \frac{1+i}{\sqrt{2}} \lambda z / \sinh \frac{1+i}{\sqrt{2}} \lambda, i=\sqrt{-1}, \lambda=\sqrt[4]{T a+R a_{V}}$. The profiles of the components of the velocity and temperature coincide, up to a multiplier, with the corresponding profiles of advective flow in a rotating fluid layer with solid horizontal boundaries described in $[4,5]$. It can be expected that the properties of the solution [14] are similar to those of the flow described in $[4,5]$. In particular, the temperature profile $\tau_{0}(z)$ and the amplitude of the pulsation velocity component $w_{x}(z)$ coincide, up to a multiplier, with the profile of the second velocity component $v_{0}(z)$. 


\subsection{A layer with free boundaries}

On the horizontal boundaries of the layer at $z= \pm 1$ :

$$
\frac{\partial v_{x}}{\partial z}=\frac{\partial v_{y}}{\partial z}=v_{z}=0, \quad T=x, \quad \frac{\partial w_{x}}{\partial z}=0
$$

The system of equations (2.6) is used to find velocity, temperature and pressure by adding the boundary conditions

$$
u_{0}^{\prime}( \pm 1)=0, \quad v_{0}^{\prime}( \pm 1)=0, \quad \tau_{0}( \pm 1)=0, \quad w_{x}^{\prime}( \pm 1)=0
$$

and the closed-loop conditions (2.8).

To find the first component of the velocity, the ordinary differential fourth-order equation (2.11) is solved using the boundary conditions (taking into account Eqs. (2.16))

$$
u_{0}^{\prime}( \pm 1)=0, \quad u_{0}^{\prime \prime \prime}( \pm 1)=1 .
$$

From the condition of flow closure (2.8) $\frac{\partial p_{h}}{\partial y}=0$ and $\frac{\partial p_{h}}{\partial x}=0$.

The second component of the velocity $v_{0}(z)$ is found using the third equation of $(2.6)$ and the corresponding conditions, Eqs. (2.16) and Eq. (2.8).

The temperature component $\tau_{0}(z)$ is found from the boundary problem (2.13).

The solution of the problem, Eq. (2.11) and Eq. (2.17), taking into account the condition of closure (2.8) has the following representation:

$$
\begin{aligned}
& u_{0}(z)=-\frac{1}{2 \lambda^{3}}\left[\frac{1+i}{\sqrt{2}} \frac{\sinh \frac{1+i}{\sqrt{2}} \lambda z}{\cosh \frac{1+i}{\sqrt{2}} \lambda}+\frac{1-i}{\sqrt{2}} \frac{\sinh \frac{1-i}{\sqrt{2}} \lambda z}{\cosh \frac{1-i}{\sqrt{2}} \lambda}\right], \\
& v_{0}(z)=-\frac{1}{2 \lambda^{3}} \sqrt{\frac{T a}{T a+R a_{V}}}\left[\frac{\sinh \frac{1+i}{\sqrt{2}} \lambda z}{\frac{1+i}{\sqrt{2}} \cosh \frac{1+i}{\sqrt{2}} \lambda}+\frac{\sinh \frac{1-i}{\sqrt{2}} \lambda z}{\frac{1-i}{\sqrt{2}} \cosh \frac{1-i}{\sqrt{2}} \lambda}\right] .
\end{aligned}
$$

Given the antisymmetric profile of the first component of the $v_{0}(z)$

$$
\begin{gathered}
\tau_{0}(z)=\operatorname{Gr} \operatorname{Pr}\left[v_{0}(z)-v_{0}(1) z\right], \\
w_{x}(z)=\operatorname{GrPr} v_{0}(z) .
\end{gathered}
$$

\section{Properties of the exact solution}

\subsection{A layer with solid boundaries}

In the absence of rotation at $T a=0$ the advective flow (2.14) coincides with [11], having one component of velocity $u_{0}(z)$. In the absence of vibration at $R_{V}=0$, the solution coincides with the description of advective flow in the rotating fluid layer with solid boundaries [4], having two components of velocity $u_{0}(z)$ and $v_{0}(z)$. The longitudinal vibration affects the advective flow like a kind of "one-dimensional" rotation. For all nonzero values of the Taylor number and the vibrational analogue of the Rayleigh number, vortex motion is formed in the layer (Fig. 2). At the same time, the effect of longitudinal vibration has a stronger effect on the $x$ th component of the velocity than on the $y$ th one (Fig. 2b, Fig. 2d). 


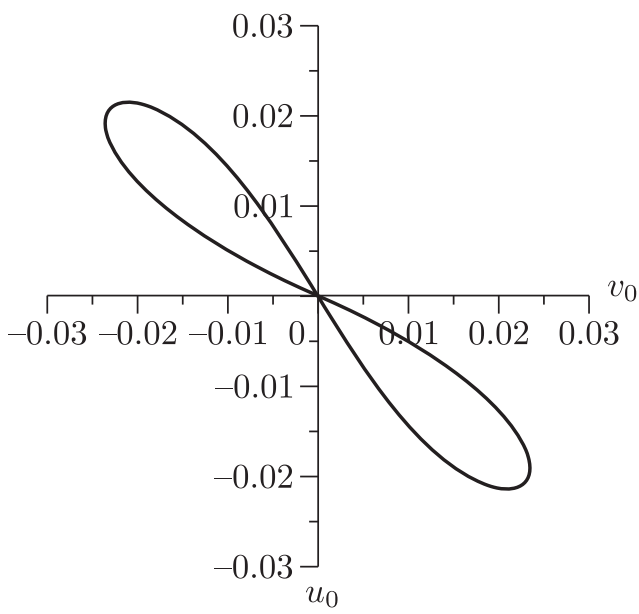

(a)

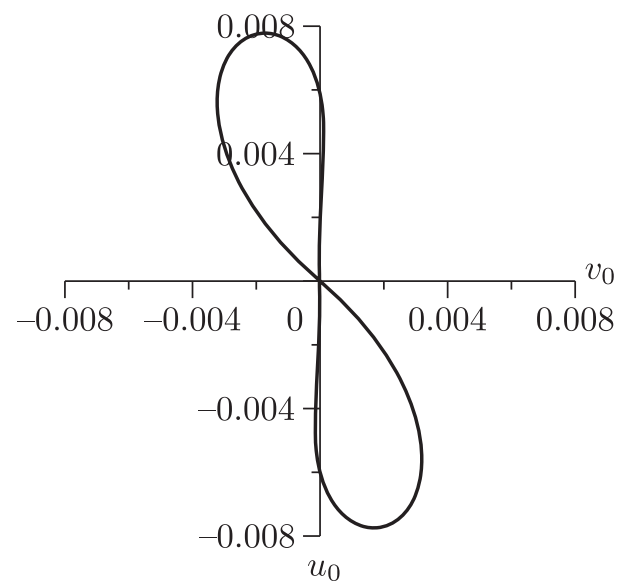

(c)

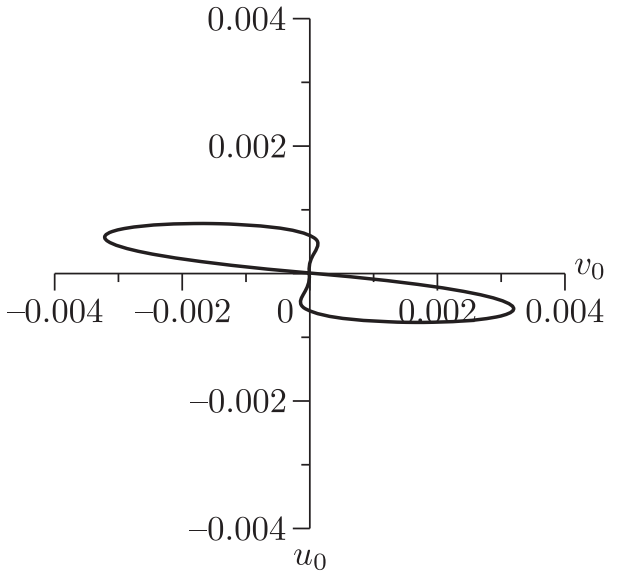

(b)

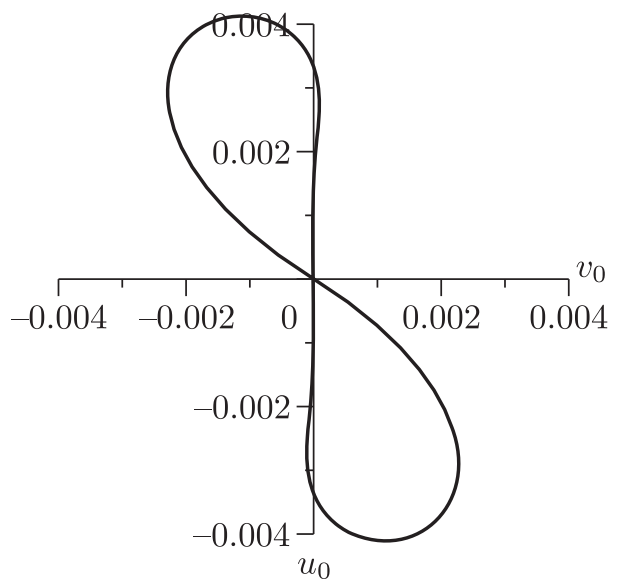

(d)

Fig. 2. Hodographs of the flow velocity at (a) $T a=100, R a_{V}=100$, (b) $T a=100, R a_{V}=10000$, (c) $T a=10000, R a_{V}=100$, (d) $T a=10000, R a_{V}=10000$.

In the absence of rotation, the advective flow has only one $x$-component of velocity. When $T a>0$ and as the Taylor numbers and (or) the vibrational analogue of the Rayleigh number grow, the maximum of the first velocity component monotonically decreases. With the growth of $T a>0$ and(or) $R a_{v}>0$ the second $y$-component of the velocity appears, the maximum of which increases to some values of the Taylor and the Gershuni numbers. A numerical study of the solution (2.14) has shown that they are determined by using an empirical formula

$$
T a \approx 98+1.07 R a_{V} .
$$

For all values of the parameters $T a$ and $R a_{V}$ the profiles of the components of the velocity $u_{0}(z), v_{0}(z)$ and temperature $\tau_{0}(z)$, as well as the amplitude of the pulsating component of the velocity $w_{x}(z)$ are antisymmetric (Fig. 3). For $T a \gg 1, R a_{V} \gg 1$, near both boundaries, boundary layers of velocity, temperature and amplitude of the pulsating component of velocity occur, and the relative thickness of the boundary layer is equal $\lambda / \sqrt{2}$. Figure $3 \mathrm{~d}$ shows the pressure graph at $x=0$. 


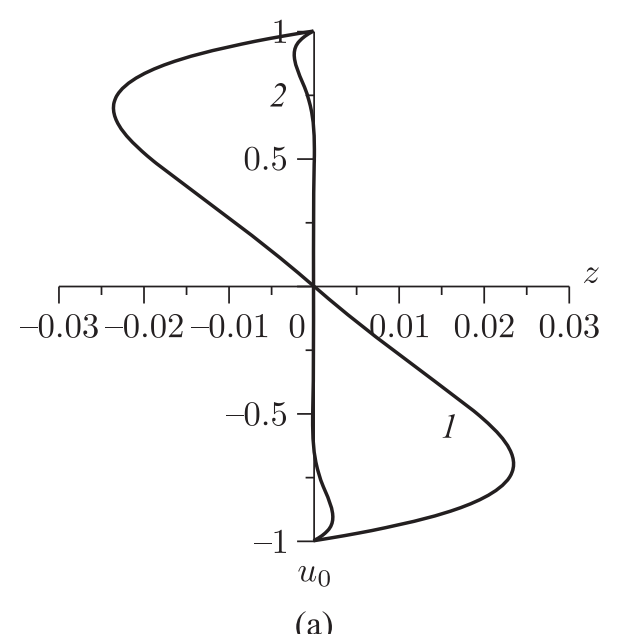

(a)

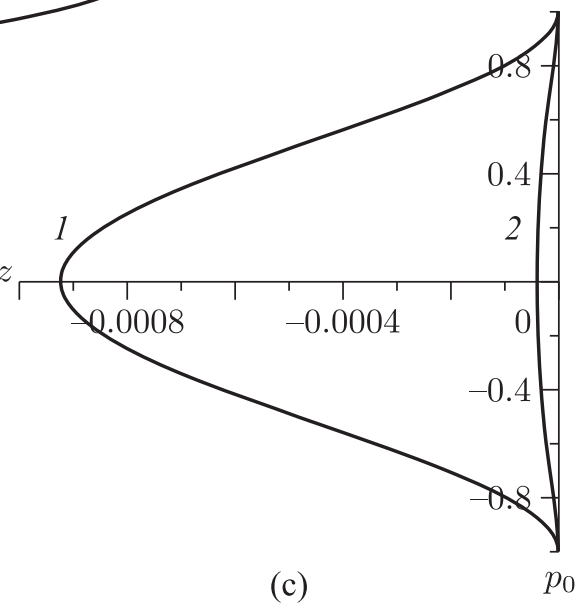

(b)

Fig. 3. The profiles of the velocity components (a) $u_{0}$, (b) $v_{0}$ and (c) pressure $p_{0}$ for $x=0$ at $1-$ $T a=100, R a_{V}=100$ and $2-T a=10000, R a_{V}=10000$.

\subsection{A layer with free boundaries}

Similar to the case with solid boundaries for all nonzero values of the Taylor number and the vibrational analogue of the Rayleigh number, a spiral motion is formed in the layer (Fig. 4). In this case, the effect of longitudinal vibration is also stronger on the $x$-th component of the velocity than on the $y$-th.

In the absence of rotation, the advective flow has only one $x$ th velocity component $u_{0}(z)$, at $T a>0$ the second $y$ th velocity component $v_{0}(z)$ appears. In this case, both components of the velocity are multidirectional along the layer. In the upper half of the layer $u_{0}(z)$ is directed from right to left, and in the lower one it is directed from left to right (Fig. 5a). On the contrary, the second velocity component in the top half is directed from left to right, and that in the bottom is directed from right to left (Fig. 5b). The temperature profile is similar to the profile of the second velocity component (Fig. 5c).

At low values of $T a$ and $R a_{V}$ the velocity component profiles are almost linear (Fig. 5), their extreme values are located at the boundaries of the horizontal fluid layer. As the values of these parameters increase, the maximum and the minimum of the second component of the velocity are shifted to the depth of the layer (Fig. 5b). Similar to the case of the layer with solid boundaries at $T a \gg 1, R a_{V} \gg 1$, near the boundaries there are boundary layers of velocity, temperature and amplitude of the pulsating velocity component, and the relative thickness of the boundary layer is equal to $\lambda / \sqrt{2}$ (Fig. 5). Figure 5 d shows the pressure graph at $x=0$. 


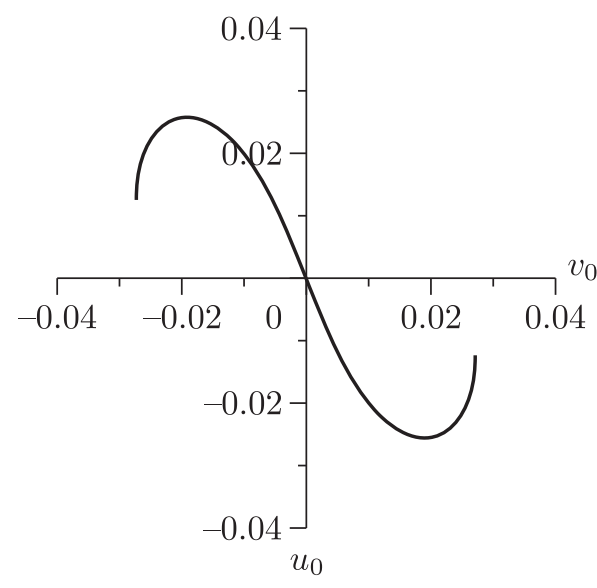

(a)

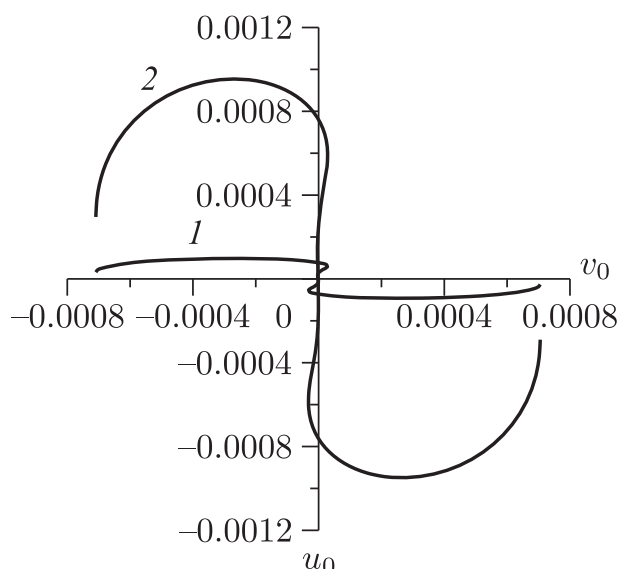

(b)

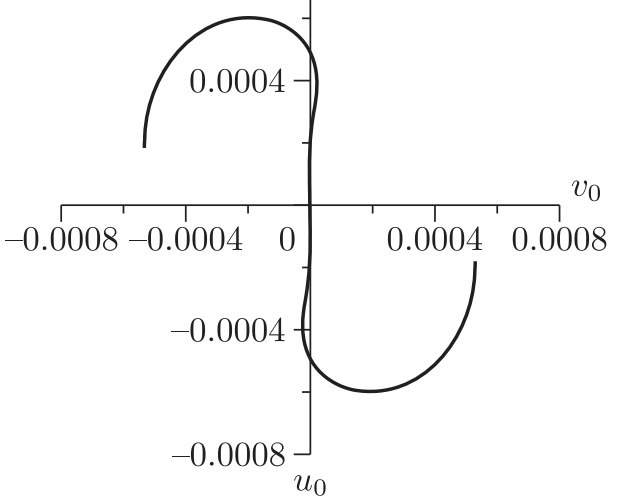

(c)

Fig. 4. Hodographs of the flow velocity at (a) $T a=100, R a_{V}=100$, (b) $T a=100, R a_{V}=10000$, (c) $T a=10000, R a_{V}=100,(d) T a=10000, R a_{V}=10000$.

\section{Conclusion}

A new exact solution of the Navier-Stokes equations in the Boussinesq approximation describing the advective flow of a rotating horizontal fluid layer in the presence of longitudinal oscillations in a rotating horizontal fluid layer for the case of solid boundaries is presented. In the absence of rotation, a known plane-parallel advective flow in a vibration field with one horizontal velocity component is obtained [11]. In the absence of vibration, a known advective flow in the rotating fluid layer is obtained too, described by two velocity components forming the vortex motion of the fluid. In the newly obtained flow, the velocity and temperature component profiles are similar to the velocity and temperature profile of the advective flow in the absence of vibration [4]. It is known [12] that the effect of rotation is largely similar to the effect of the magnetic field. It can be argued that the effect of longitudinal vibration on the advective flow is somewhat similar to the effect of rotation. The influence of the longitudinal vibration in this case can be called "one-dimensional" rotation.

A new exact solution of the Navier-Stokes equations in the Boussinesq approximation describing advective flows of a rotating horizontal fluid layer in the presence of longitudinal oscillations in a rotating horizontal fluid layer for the case of free boundaries is presented. The flow has two horizontal velocity components. The velocity and temperature profiles of the 


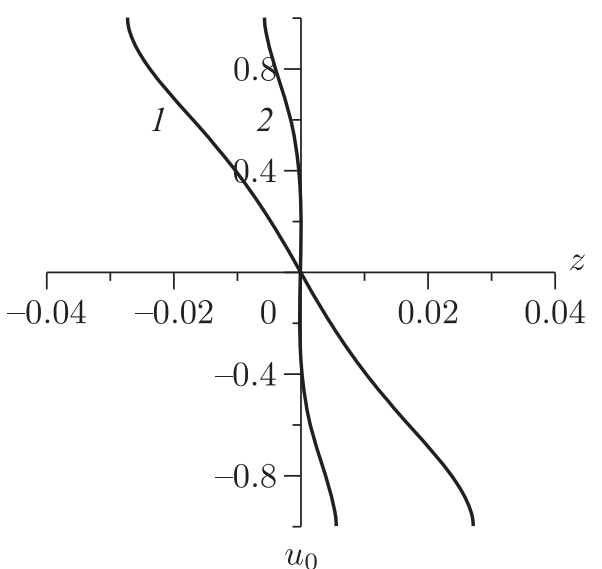

(a)

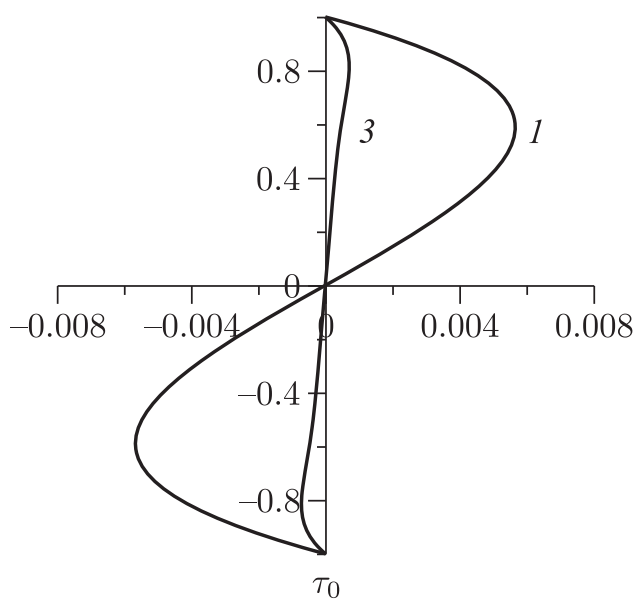

(c)

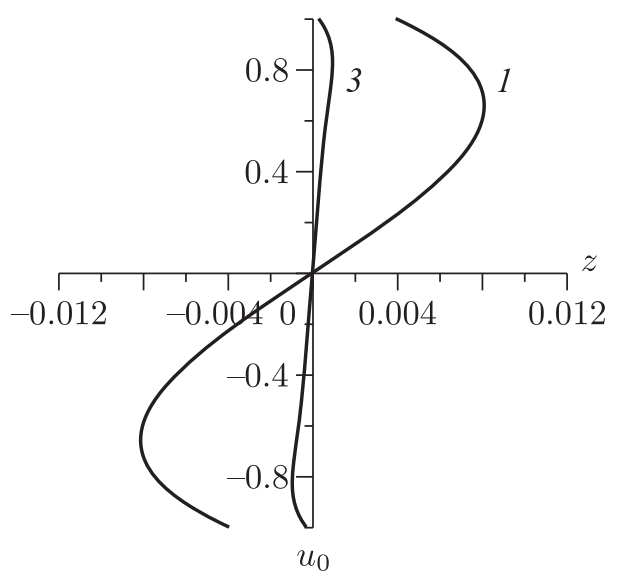

(b)

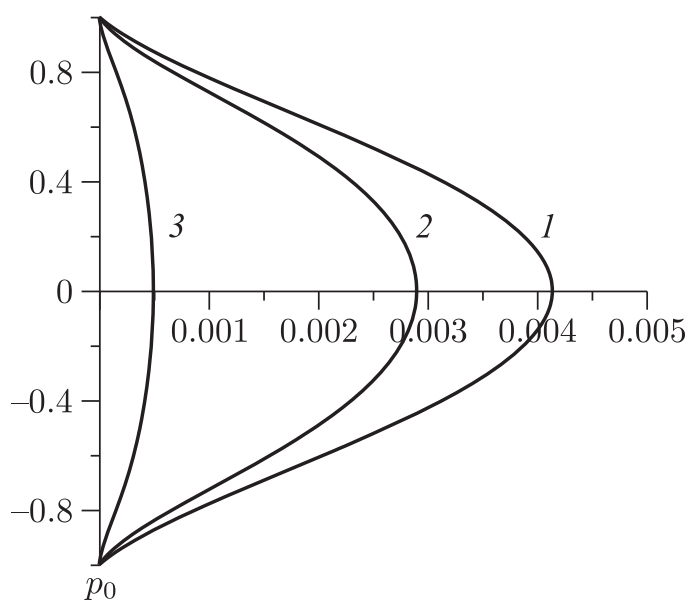

(d)

Fig. 5. The profiles of the velocity components (a) $u_{0}$, (b) $v_{0}$, (c) $\tau_{0}$ and (d) $p_{0}$ for $x=0$ at $1-T a=10$, $2-T a=1000,3-T a=10000$.

advective flow as well as for the case of rigid boundaries are antisymmetric. A spiral movement is formed, with fast rotation and (or) high-frequency longitudinal vibration near the boundaries of the layer, and thin boundary layers of velocity and temperature are formed.

\section{References}

[1] Andreev, V.K., The Birikh Solution of the Convection Equations and Some Its Generalizations, Preprint No. 1-10, Krasnoyarsk: Inst. Comput. Math. SB RAS, 2010 (Russian).

[2] Anisimov, I. A. and Birikh, R. V., Hydrodynamic Instability of Vibration Advective Flow in Microgravity, in Vibrational Effects in Hydrodynamics: Vol.1, D. V. Lyubimov (Ed.), Perm: Perm. Gos. Univ., 1998, pp. 17-24 (Russian).

[3] Aristov, S. N. and Prosviryakov, E. Yu., A New Class of Exact Solutions for Three-Dimensional Thermal Diffusion Equations, Theor. Found. Chem. Eng., 2016, vol. 50, no. 3, pp. 286-293; see also: Teoret. Osnovy Khim. Tekhnolog., 2016, vol. 50, no. 3, pp. 294-301. 
[4] Aristov, S. N. and Schwarz, K. G., Vortex Flows of Advective Nature in a Rotating Fluid Layer, Perm: Perm. Gos. Univ., 2006 (Russian).

[5] Aristov, S. N. and Shvartz, K. G., Stability of the Advective Flow in a Rotating Horizontal Fluid Layer, Fluid Dynam., 1999, vol.34, no.4, pp.457-464; see also: Izv. Ross. Akad. Nauk. Mekh. Zidk. Gaza, 1999, vol. 34, no.4, pp. 3-11.

[6] Bardin, B. S. and Panev, A. S., On the Motion of a Body with a Moving Internal Mass on a Rough Horizontal Plane, Russian J. Nonlinear Dyn., 2018, vol. 14, no. 4, pp. 519-542.

[7] Birikh, R. V. and Katanova, T. N., Effect of High-Frequency Vibrations on the Stability of Advective Flow, Fluid Dynam., 1998, vol.33, no.1, pp.12-17; see also: Izv. Ross. Akad. Nauk. Mekh. Zidk. Gaza, 1998, no. 1, pp. 16-22.

[8] Birikh, R. V. and Katanova, T. N., On Stabilization of Advective Flow by Transverse Vibrations, in Vibrational Effects in Hydrodynamics: Vol. 1, D. V. Lyubimov (Ed.), Perm: Perm. Gos. Univ., 1998, pp. 25-37 (Russian).

[9] Birikh, R. V., Vibrational Convection in a Plane Layer with a Longitudinal Temperature Gradient, Fluid Dynam., 1990, vol.25, no.4, pp.500-503; see also: Izv. Akad. Nauk SSSR Mekh. Zhidk. Gaza, 1990, no. 4, pp. 12-15.

[10] Borisov, A. V. and Mamaev, I. S., Isomorphisms of Geodesic Flows on Quadrics, Regul. Chaotic Dyn., 2009, vol. 14, nos. 4-5, pp. 455-465.

[11] Gershuni, G.Z. and Zhukhovitskii, E. M., Plane-Parallel Advective Flows in Vibrational Field, J. Eng. Phys., 1989, vol.56, no. 2, pp. 238-242; see also: Inzh.-Fiz. Zh., 1989, vol.56, no. 2, pp. 238242.

[12] Gershuni, G.Z. and Zhukhovitskii, E. M., Convective Stability of Incompressible Liquid, Jerusalem: Wiley, 1976.

[13] Gershuni, G.Z., Zhukhovitskii, E.M., and Nepomnyashchii, A. A., Stability of Convective Flows, Moscow: Nauka, 1989 (Russian).

[14] Gershuni, G.Z. and Lyubimov, D. V., Thermal Vibrational Convection, New York: Wiley, 1998.

[15] Hudoba, A., Molokov, S., Aleksandrova, S., and Pedcenko, A., Linear Stability of Buoyant Convection in a Horizontal Layer of an Electrically Conducting Fluid in moderate and High Vertical Magnetic Field, Phys. Fluids, 2016, vol. 28, no. 9, 094104, 15 pp.

[16] Kaddeche, S., Henry, D., and Benhadid, H., Magnetic Stabilization of the Buoyant Convection between Infinite Horizontal Walls with a Horizontal Temperature Gradient, J. Fluid Mech., 2003, vol. 480, pp. 185-216.

[17] Ostroumov, G. A., Free Convection under the Condition of the Internal Problem (NACA-TM-1407, Rept-4281), Washington, D.C.: NASA, 1958. 